\title{
Professor Xiaoyan Wang used Yinhuo Decoction to Treat Yang Insomnia from not Entering Yin Type
}

\author{
Yihan Liu', Linlin Yang1, Xiaoyan Wang2* \\ ${ }^{1}$ Shaanxi University of Chinese Medicine, Xianyang 712046, Shaanxi Province, China \\ ${ }^{2}$ Xi'an Hospital of Traditional Chinese Medicine, Xi'an 710021, Shaanxi Province, China \\ *Corresponding author: Xiaoyan Wang, wxiaoyan3046@163.com
}

\begin{abstract}
Yinhuo decoction is recorded by Shiduo Chen, a doctor in the Qing Dynasty, in the Dialectical Record. It is mainly used to treat "tonsillitis with yin asthenia." Its pathogenesis is deficiency of kidney Yin and Yang-function insufficiency of kidney Zang, resulting in vacuity heat floating upward. Professor Xiaoyan Wang, the tutor, believes that the general pathogenesis of insomnia is that Yang does not enter Yin. The application of Yinhuo Decoction in clinic to treat insomnia is quite effective.
\end{abstract}

Keywords: Yinhuo Decotation; Insomnia; Sleepless; Yang does not enter the Yin

Publication date: September 2021; Online publication: September 30, 2021

\section{Compatibility characteristics of Yinhuo Decoction}

Original recipe of Yinhuo Decoction: Radix Rehmanniae Preparata 150g, Morinda officinalis 50g, Poria $\operatorname{cocos} 25 \mathrm{~g}$, Radix Ophiopogonis 50g, northern schisandra 10g. In the prescription radix rehmanniae preparata is used more as principal drug, filling the true Yin. Shiduo Chen believes that "the medicine for tonifying Yang can be used less to work, while the medicine for tonifying Yin must be used more to take effect". Yang Qi is mainly promoted by its nature, so the amount can rise with its nature, and the amount of Yin medicine is small, which is difficult to release ${ }^{[1]}$. Morinda officinalis is a adjuvant drug, which warms the kidney and helps Yang, supplemented by Radix Ophiopogonis. It can not only clear the upper coke and floating heat, but also nourish yin and moisten the lungs, reflecting the coexistence of gold and water. Poria cocos strengthens the spleen, can channel the water, coordinate the gasification of the triple energizers, run the central axis, help the floating Yang heat return to the lower energizer Kangua, and schisandra chinensis converges the Qi of the five directions to the groundwater Yin, enhancing the source of life. The whole recipe aims to return fire to the origin, treat Yin and Yang together, strengthen local Qi, nourish the yin with sour and sweet, and increase the source of life. $\mathrm{Ke} \mathrm{Li}$, a famous modern and old traditional Chinese medicine, is good at using Yinhuo Decoction plus Radix Asparagi and cinnamon to treat many diseases such as mouth sore, epistaxis and headache caused by kidney yin deficiency and ministerial fire flame upward ${ }^{[2-3]}$.

\section{Understanding of insomnia by doctors of past dynasties}

Insomnia is called "no sleeping" and "no sleep" in the internal classic. It is considered that its pathogenesis is that evil Qi is attracted to the viscera and defensive Qi doesn't enter Yin. In the book In the Scriptures, sleepless is called "insomnia," which puts forward that "if the gallbladder is cold, there will be no sleep" and "if the heart is weak, there will be no sleep." It is considered that insomnia is related to cold gallbladder and deficiency of heart Qi. Yuanfang Chao believes that insomnia is due to the patient's serious disease, 
physical deficiency, decline of viscera and vitality, deficiency of construction-yin and disharmony of construction-defense. Simiao Sun used Wendan Decoction to treat insomnia in "Valuable Prescriptions for Emergency." He believed that the cause of insomnia was cold and took tranquilizing drugs. Shiduo Chen believes that insomnia is caused by heart-kidney imbalance.

\section{Tutor's understanding of the pathogenesis of insomnia}

Under the theoretical guidance of the Internal Classic, the tutor analyzed from the "Qi monism" that the main reason for insomnia is that "Yang does not enter Yin", that is, Yang is on the top and cannot hide in the kidney water. This kind of Yang that cannot dive is not a normal Yang. It is the dysfunction of the functions of Qi in the human viscera, resulting in a series of "dryness heat and fire evil," which disturbs the mind, so it's hard to sleep. There are several types that cause Yang Qi to not be hidden.

(1) The liver Qi of reverting Yin does not rise, and the A gallbladder do not fall: The normal ascending and descending movements of A wood and B wood make the liver Qi of reverting Yin rise slowly and orderly, and the Qi of lesser Yang gallbladder meridian fall to the right. If the liver Qi rises and becomes weak, the A gallbladder will not fall, the gallbladder fire will rise, and will become phase fire, disturbing the state of mind and will not sleep. Liver Qi is budding Qi, and liver depression is mostly caused by weak start-up, so liver Qi cannot rise gently and orderly. Just exercise liver Qi to help liver rise and relieve liver depression. In clinic, tutors often use Ramulus cinnamomian, Fructus evodiae, low-dose Radix Astragali seu Hedysari, Radix Bupleuri and so on to raise budding Qi. If the patient has excessive liver depression, the dosage of Radix Bupleuri can be increased, Herb Menthae, Radix Auckland and Radix Curcumae are used to dispel liver depression. Liver belongs to Yin in physique and Yang in function. Radix Angelicae Sinensis and Cornus are used to supplement the liver physique and help the liver function.

(2) The middle earth is damaged and the central axis is not transported: The Heart Source of the Four Saints says that "The spleen is F earth, and the spleen is mainly ascending by greater Yin, while stomach is E earth, mainly descending with Yangming ${ }^{[4]}$." The central axis of spleen and stomach is damaged, the function of raising clear and lowering turbidity is lost, the F earth does not rise, phlegm dampness and blood stasis block the middle energizer, and depression turns into fire, resulting in insomnia. Medicine uses high-dose Radix Astragali seu Hedysari, Rhizoma Atractylodis Macrocephalae and so on to turn the central axis, thicken earth Qi.

(3) The Qi of lung and stomach does not drop: The Qi on the right road does not fall, blocking the functioning of Qi. Yang Qi cannot be hidden in the Yin of groundwater. Yang Qi floats on the top, resulting in dryness, heat and fire evil. The patient is difficult to sleep, feels upset, etc. The Qi of the lung and stomach does not fall, which will also lead to the loss of conduction in the large intestine, and the stagnation of heat in the intestine will disturb the mind. Heart-Draining Decoction and winetreated rhubarb are used to reduce heart fire and Qi of lung and stomach.

(4) Deficiency of kidney Yin and kidney Yang: After middle age, the patient had deficiency of kidney Yin and kidney Yang, and could not collect and store Yang Qi. The Dragon thunder fire disturbed the mind and couldn't sleep. In the clinic, the Marrow-Sealing Elixir is often used to subdue Yang and seal the marrow, the Radix Rehmanniae Preparata nourishes the true Yin, and the achyranthes reduces the right way to enhance the source of life.

\section{Examples of medical records}

Patient Yang, female, 50 years old, complained of poor sleep in recent three years, falling asleep well but awakening early, which mostly ranging from 4:00 to 5:00 in the morning, difficult to fall asleep again after 
waking up, dreamy, mostly nightmares, occasional dizziness, headache, dry lips, more thinking, poor mood, tired work, eating well, dry stool, defecating once every 4-5 days, and urination well. She was always in good health. The menstrual volume was less and the cycle was irregular in recent one year. Diagnosis was: Sleepless. Syndrome differentiation was: Deficiency of kidney Yin and vacuity heat floating upward. Prescription was: Yinhuo Decoction combined with Cinnamon Twig, Licorice, Dragon Bone, and Oyster Shell Decoction plus or minus. Radix Rehmanniae Preparata 30g, Morinda officinalis 15g, Poria cocos 30g, Radix Ophiopogonis 10g, Schisandra chinensis 6g, Radix Asparagi 10g, cinnamon 6g, Radix Angelicae Sinensis 30g, Ramulus Cinnamomiand 15g, Radix Paeoniae Rubra 30g, crude dragon bone 30g, raw oyster shell 30g, roasted Radix Glycyrrhizae 15g, raw Rhizoma Atractylodis Macrocephalae 60g, Polyporus Umbellatus $10 \mathrm{~g}$ and fried Semen Ziziphi Spinosae 10g. Totally there was 7 doses, taking 1 dose per day, decocted with water.

Second diagnosis: The patient complained that after taking the medicine, the symptoms of early waking were better than before, and she still had nightmares, dry lips and dry stool. In the original prescription, the dosage of radix rehmanniae preparata was increased to $45 \mathrm{~g}$, added wine-treated rhubarb $10 \mathrm{~g}$, Fructus Mume $10 \mathrm{~g}$, and followed by 7 doses.

Dialectical analysis was: The patient was usually tired and in menopause. The sky was exhausted, the liver and kidney were insufficient, the water was shallow, the Dragon thunder was burnt on the fire, and she was always dizzy and headache. Reverting Yin subsides, the A gallbladder turn up against the fire and disturb the spirit. Therefore, seeing poor sleep, thinking and having poor mood, we could use Yinhuo Decoction to lead the fire to the original, guide the dragon to the sea and strengthen the source of life. Ramulus Cinnamomiand helps the sprout, the Os Draconis and oyster secure and contain the essence of the kidney and astringe the vitality. Radix Angelicae Sinensis and Semen Ziziphi Spinosae benefit liver yin and liver blood. 60g raw Rhizoma Atractylodis Macrocephalae mediate the middle Qi, that is, the meaning of "spleen governs the dispersion of essence and transportation." Radix Paeoniae Rubra and roasted Radix Glycyrrhizae take the meaning of Peony and Licorice Decoction to reduce the upper A gallbladder. In the second diagnosis, the sleep of the patient was better than before, and there were still nightmares so the dosage of the radix rehmanniae preparata was increased. And her stool was dry, considering that the patient combined reverting Yin centralization, thermalization and Yang brightness visceral heat on the basis of the dragon thunder fire burnt. Thus, add Fructus Mume, wine-treated rhubarb to collect to drop off phase fire, close reverting Yin, decline Yang brightness.

\section{Disclosure statement}

The author declares no conflict of interest.

\section{References}

[1] Chen SD, 2011, New Edition of Materia Medica. Beijing: China Traditional Chinese Medicine Science and Technology Press, 23.

[2] Li K, 2019, Experience Album of Li Ke, an Old Doctor of Traditional Chinese Medicine, with Acute, Critical and Difficult Diseases. Shanxi Science and Technology Publishing House, 7.

[3] Gao F, 2007, Clinical Application Experience of Yinhuo Decoction. Journal of Shandong University of Traditional Chinese Medicine, (06): 483-484.

[4] Huang YY, 2009, Four Shengxinyuan. Annotator: Qianxi Sun. Beijing: China Press of Traditional Chinese Medicine. 